\title{
A CONTINUOUS GROUP RELATED TO VON SEIDEL'S OPTICAL THEORY.
}

BY DR. ARTHUR C. LUNN.

(Read before the Chicago Section of the American Mathematical Society, April 17, 1908.)

THE determination of the varions aberrations of an axially symmetric optical instrument according to the method systematized by Petzval and von Seidel rests on the computation of certain power series whose coefficients are functions of what may be called the paraxial magnitudes of the system. These are the quantities which describe what would be the course of the rays of light through the ideal instrument, to which the actual instrument is an approximation, and which was defined by Abbe* as producing an exact collineative transformation of the object into the image. This ideal transformation coincides with the classic first approximation of geometric optics, a general analytic representation of which was given by Gauss, $\dagger$ together with a method of computation using continued fractions. A set of formulas more convenient in practice was introduced by von Seidel, $\$$ using as defining coordinates of a ray in a meridian plane the optical height, or distance of the ray from any point of the axis, measured along the normal to the latter, and the angle, or in the ideal collineation the tangent of the angle, between the ray and the axis.

If these coordinates be called $x, \theta$ respectively, von Seidel's paraxial equations are equivalent to

$$
\Delta \mu \theta=x k \Delta \mu, \quad \Delta x=0, \quad D \theta=0, \quad D x=-t \theta,
$$

where $\mu$ is the refractive index of a medium, $k$ the curvature of a refracting spherical surface, $t$ the distance of transmission through a homogeneous medium; $\Delta, D$ denote the changes corresponding respectively to refraction at a surface where $\mu$ changes and to propagation through a medium of constant $\mu$ separating two consecutive surfaces. It will be shown here that these equations may be viewed as transformations generat-

\footnotetext{
* Czapski-Eppenstein, Theorie der optischen Instrumente nach Abbe, chap. ii.

$†$ Dioptrische Untersuchungen, Ges. Werke, V, p. 243.

$\ddagger$ Astr. Nach., Nos. 1027-1029 (April, 1855).
} 
ing a certain three-parameter group, whose properties thus admit of optical interpretation. In particular the determination of the complete system of invariants seems to mark the exact scope of possible application of Abbe's concept of "optical invariant" in the formulation of optical computations.

Let $\mu \theta=u$, then the transformations and their generating differentiators are

$$
\begin{aligned}
& u^{\prime}=u+\alpha x, \quad x^{\prime}=x, \quad U_{1}=x \frac{\partial}{\partial u}, \quad(\alpha=k \Delta \mu) \\
& x^{\prime}=x+\beta u, \quad u^{\prime}=u, \quad U_{2}=u \frac{\partial}{\partial x}, \quad\left(\beta=-\frac{t}{\mu}\right)
\end{aligned}
$$

each of which defines a one-parameter group in the variables $x, u$, the respective parameters $\alpha, \beta$ being arbitrary because the equations apply to systems having arbitrary curvatures and axial spacing of the refracting surfaces.

The first group relates to successive refractions at a set of surfaces in contact, and corresponds to the ordinary theory of thin lenses close together. For example, the additive property of the focal powers of such lenses is the interpretation of the additive combination of the parameters $\alpha$ in the compounding of the transformations, while the invariance of $x$ means simply that the primitive and refracted rays intersect at the refracting surface.

The second group relates to the optical effect of media stratified in parallel planes. The invariance of $u$ means that such a system has no focal power according to the definition of Gauss, and the additive combination of parameters corresponds to the foreshortening effect of each stratum according to its thickness and refractive index.

The dual character of the two transformations under simultaneous interchange of $x, u$ and $\alpha, \beta$ was recognized by von Seidel in his use of odd and even subscripts respectively for refracting surfaces and intervening media. It suggests its own phrasing for various statements. For instance, a principal focus is a point where $x=0$ on a ray transformed from a primitive ray having $u=0$, or parallel to the axis.

The successive commutators formed from $U_{1}$ and $U_{2}$ are

$$
\begin{gathered}
\left(U_{1} U_{2}\right)=U_{3}=x \frac{\partial}{\partial x}-u \frac{\partial}{\partial u}, \quad\left(U_{1} U_{3}\right)=-2 U_{1}, \\
\left(U_{2} U_{3}\right)=2 U_{2} .
\end{gathered}
$$


There is thus generated a three-parameter group whose equations may be obtained* by integration of

$$
\frac{d x^{\prime}}{d t}=\lambda_{2} u^{\prime}+\lambda_{3} x^{\prime}, \quad \frac{d u^{\prime}}{d t}=\lambda_{1} x^{\prime}-\lambda_{3} u^{\prime},
$$

giving

$$
\begin{aligned}
& x^{\prime}=A_{1} \lambda_{2} e^{\omega t}+A_{2} \lambda_{2} e^{-\omega t}, \\
& u^{\prime}=A_{1}\left(\omega-\lambda_{3}\right) e^{\omega t}-A_{2}\left(\omega+\lambda_{3}\right) e^{-\omega t}, \\
& \omega^{2}=\lambda_{3}^{2}+\lambda_{1} \lambda_{2},
\end{aligned}
$$

where the constants $A$ are to be determined in terms of the initial coordinates $x, u$. A convenient final form, containing the three parameters $a, b, c$ is

$$
x^{\prime}=x C+(c x+b u) S, \quad u^{\prime}=u C+(a x-c u) S,
$$

where $C=\cosh \tau, S=\sinh \tau / \tau, \tau^{2}=c^{2}+a b$, and $a=\lambda_{1} t$, $b=\lambda_{2} t, c=\lambda_{3} t$. The groups of $U_{1}$ and $U_{2}$ individually are obtained by putting respectively $b=c=\tau=0, a=\alpha$, and $a=c=\tau=0, b=\beta$.

The combination of two transformations such as (4) with parameters $(a, b, c)$ and $\left(a^{\prime}, b^{\prime}, c^{\prime}\right)$ is equivalent to a single such transformation with parameters $\left(a^{\prime \prime}, b^{\prime \prime}, c^{\prime \prime}\right)$ determined by

$$
\begin{aligned}
C^{\prime \prime}+c^{\prime \prime} S^{\prime \prime} & =\left(C^{\prime}+c^{\prime} S^{\prime}\right)(C+c S)+a b^{\prime} S^{\prime} S, \\
b^{\prime \prime} S^{\prime \prime} & =b S\left(C^{\prime}+c^{\prime} S^{\prime}\right)+b^{\prime} S^{\prime}(C-c S), \\
C^{\prime \prime}-c^{\prime \prime} S^{\prime \prime} & =\left(C^{\prime}-c^{\prime} S^{\prime}\right)(C-c S)+a^{\prime} b S^{\prime} S, \\
a^{\prime \prime} S^{\prime \prime} & =a S\left(C^{\prime}-c^{\prime} S^{\prime}\right)+a^{\prime} S^{\prime}(C+c S),
\end{aligned}
$$

which may be replaced by

$$
\begin{aligned}
C^{\prime \prime} & =C C^{\prime}+S S^{\prime}\left\{c c^{\prime}+\frac{1}{2}\left(a b^{\prime}+a^{\prime} b\right)\right\}, \\
c^{\prime \prime} S^{\prime \prime} & =c S C^{\prime}+c^{\prime} S^{\prime} C+\frac{1}{2} S S^{\prime}\left(a b^{\prime}-a^{\prime} b\right), \\
b^{\prime \prime} S^{\prime \prime} & =b S C^{\prime}+b^{\prime} S^{\prime} C+S S^{\prime}\left(b c^{\prime}-b^{\prime} c\right), \\
a^{\prime \prime} S^{\prime \prime} & =a S C^{\prime}+a^{\prime} S^{\prime} C+S S^{\prime}\left(c a^{\prime}-c^{\prime} a\right) .
\end{aligned}
$$

Here the first equation determines the set of possible values of the auxiliary $\tau^{\prime \prime}$, then $a^{\prime \prime}, b^{\prime \prime}, c^{\prime \prime}$ are given by the last three

* Lie-Engel, Theorie der Transformationsgruppen, I, p. 70. 
equations; and a direct computation shows that if $\tau^{2}=c^{2}+a b$ and ${\tau^{\prime 2}}^{2}=c^{\prime 2}+a^{\prime} b^{\prime}$ then also ${\tau^{\prime \prime}}^{2}=c^{\prime \prime 2}+a^{\prime \prime} b^{\prime \prime}$, verifying the group property. The solution fails only if $S^{\prime \prime}=0$, which occurs when $\tau^{\prime \prime}$ has the form $2 n \pi i$ where $n$ is an integer not zero. With $\tau$ in the neighborhood of such a value it is more convenient to use $a / \tau, b / \tau, c / \tau$ as parameters. It should be noticed that some complex values of the parameters give real transformations, and that if $(a, b, c)$ and $\left(a^{\prime}, b^{\prime}, c^{\prime}\right)$ are real numbers, still $\left(a^{\prime \prime}, b^{\prime \prime}, c^{\prime \prime}\right)$ are not necessarily such. Moreover, $(a, b, c)$ may be real but $\tau$ pure imaginary, if $c^{2}+a b$ is negative.

The group of $U_{3}$ alone, obtained by putting $a=b=0$, $c=\tau$, has the form

$$
x^{\prime}=x e^{\tau}, \quad u=u e^{-\tau},
$$

where the parameters combine additively and $x u$ is invariant.

The general transformation (4) can be built up in the form $T_{2} T_{1} T_{2}^{\prime}$, where each $T_{i}$ is a transformation of the one-parameter group of $U_{i^{*}}$ For, if the factors have parameters $\beta, \alpha, \beta^{\prime}$ respectively, the identification rests upon the solution of the equations

$$
\begin{gathered}
1+\beta \alpha=C+c S, \quad \alpha=a S, \quad 1+\beta^{\prime} \alpha=C-c S, \\
\beta+\beta^{\prime}+\beta \beta^{\prime} \alpha=b S .
\end{gathered}
$$

The first three give

$$
\alpha=a S, \quad \beta=(C-1+c S) / a S, \quad \beta^{\prime}=(C-1-c S) / a S,
$$

and these values satisfy the fourth equation also, because of the relation $\tau^{2}=c^{2}+a b$. An alternative factorization is the dual form $T_{1} T_{2} T_{1}^{\prime}$, with parameters $\alpha, \beta, \alpha^{\prime}$, obtained by interchange of $\alpha$ and $\beta$ and also of $a$ and $b$.

The first form interprets the general transformation in terms of a single refracting surface as giving the relation of the coordinates of a refracted ray at distance $t^{\prime}$ behind the surface to those of the primitive ray at distance $t$ in front of the surface. A special case of interest is where $x^{\prime}$ is independent of $u$, or $\beta+\beta^{\prime}+\beta \beta^{\prime} \alpha=0$. This is equivalent to $\mu / t+\mu^{\prime} / t^{\prime}=$ $\left(\mu^{\prime}-\mu\right)_{i} r$, the ordinary equation of conjugate distances with respect to the surface. Such transformations occur for instance when the successive positions of a ray traversing any system are located with respect to the planes of the aperture diaphragm and its successive images. 
The second form interprets the general transformation in terms of two separated refracting surfaces, and gives the relation of the coordinates of the primitive and refracted rays at points in the first and third media in contact with the surfaces; giving thus an analogy to the theorem of Gauss that any system is in the first approximation equivalent to a single thick lens. The special case, dual to the one mentioned in the preceding paragraph, is where $u^{\prime}$ is independent of $x$, or $\alpha+\alpha^{\prime}+\alpha \alpha^{\prime} \beta=0$. This is equivalent to the condition that the second principal focus of the first surface coincides with the first of the second surface, so that the lens is "afocal" or telescopic.

The three-parameter group in two variables thus far considered has no invariant other than a constant. But in the estimation of certain optical errors, such as astigmatism and distortion, which depend on the location of the apertures limiting the pencils of rays, it is necessary to consider not only the systems of rays proceeding from the object and transformed into those converging upon the images formed by the successive refractions, but also a fictitious system of rays proceeding from the "entrance pupil" or initial aperture diaphragm, real or virtual, and refracted into those converging upon its successive images in the same optical system. If the rays of this second system be specified by coordinates $v, y$, then these will be variables transformed cogrediently with $u, x$, by equations similar to (4) with the same parameters, belonging thus to a three-parameter group in the four variables generated by

$$
\begin{gathered}
U_{1}=x \frac{\partial}{\partial u}+y \frac{\partial}{\partial v}, \quad U_{2}=u \frac{\partial}{\partial x}+v \frac{\partial}{\partial y}, \\
U_{3}=\left(U_{1} U_{2}\right)=x \frac{\partial}{\partial x}+y \frac{\partial}{\partial y}-u \frac{\partial}{\partial u}-v \frac{\partial}{\partial v},
\end{gathered}
$$

so that a fundamental system will consist of a single proper invariant.

To find its form, let $\rho=u x+v y, \sigma=u y-v x$. Then the $U$ 's expressed in terms of the variables $(x, y, \rho, \sigma)$ are

$$
\begin{gathered}
U_{1}=\left(x^{2}+y^{2}\right) \frac{\partial}{\partial \rho}, \quad U_{3}=x \frac{\partial}{\partial x}+y \frac{\partial}{\partial y}, \\
U_{2}=\frac{1}{\left(x^{2}+y^{2}\right)}\left\{(\rho x+\sigma y) \frac{\partial}{\partial x}+(\rho y-\sigma x) \frac{\partial}{\partial y}+\left(\rho^{2}+\sigma^{2}\right) \frac{\partial}{\partial \rho}\right\}
\end{gathered}
$$


whose form shows directly that an invariant must be independent of $x, y, \rho$, and therefore a function of $\sigma$ only.

If $u, x$ belong to a ray passing through the margin of the image and the axial point of the diaphragm, and $v, y$ similarly to a ray passing through the center of the image and the edge of the diaphragm, a geometric construction shows that the expression $u y-v x$ is equivalent to the product of refractive index, lateral radius of image, and tangent of angular semi-aperture of central pencil. The invariant $\sigma$ is therefore identical with the expression which occurs in the well-known equation pointed out in a special case by Lagrange, but first found as general by Helmholtz.* The result here shows that this is essentially the only relation expressible in terms of invariants which gives a general property of the paraxial transformation.

Chicago, Ill.,

April, 1909.

\section{SHORTER NOTICES.}

Analytische Geometrie der Ehene. By C. Runge. Leipzig, B. G. Teubner, 1908. 198 pp.

SHALl we regard elementary analytic geometry, the analytic geometry we teach in a first course to freshmen or sophomores, as a body of doctrine with which it is useful for the student to become acquainted, or shall we rather regard it as an instrument with whose use he is to be made so familiar that he shall always be ready to employ it even in a quite new problem? This is a question which every teacher of analytic geometry and every writer of a text-book on the subject is called upon to face. Upon its answer the nature of the textbook written or selected for use will depend. Few persons, it is true, would go to such an extreme as to adopt without qualification either of the views above referred to. Those who regard it as their main object to inculcate a beautiful and important doctrine would deem it essential that the student gain the power of making the application of the general theorems learned to concrete cases, and the teacher who regards elementary analytic geometry primarily as a method whose use is to be taught would not neglect the opportunity of explaining

* Handbuch der physiologischen Optik, 1. Auflage, p. 50. 